\title{
某些射影平坦的 $(\boldsymbol{\alpha}, \boldsymbol{\beta})$ - 度量*
}

\author{
沈一兵 ${ }^{* *}$ 赵例俐
}

(浙江大学数学系, 杭州 310028)

摘要 考虑了一类具有如下形式的 Finsler 度量:

$$
F=\alpha+\varepsilon \beta+\frac{2 k \beta^{2}}{\alpha}-\frac{k^{2} \beta^{4}}{3 \alpha^{3}},
$$

其中 $\alpha=\sqrt{a_{i j} y^{i} y^{j}}$ 是一个 Riemann 度量, $\beta=b_{i} y^{i}$ 是一个 1 - 形式, $\varepsilon$ 和 $k \neq 0$ 是 常数. 得到了 $F$ 为局部射影平坦的充要条件, 给出了非平凡特解, 并且证明了 具有常旗曲率的这种射影平坦 Finsler 度量是局部 Minkowski 度量.

\section{关键词 射影平坦 $\operatorname{Finsler}(\boldsymbol{\alpha}, \boldsymbol{\beta})$ - 度量 旗曲率}

\section{1 引言}

Finsler 几何中的一个基本问题是研究 Finsler 度量在开区域 $U \subset \mathbb{R}^{n}$ 中射影 平坦的特征. Finsler 度量在 $U$ 上射影平坦是指其测地线为直线, 这是 Hilbert 第 4 问题的一般情形 ${ }^{[1]} .1903$ 年, Hamel ${ }^{[2]}$ 证明了度量 $F=F(x, y)$ 在 $U$ 上射影平 坦的充要条件为

$$
F_{x^{i} y^{j}} y^{i}=F_{x^{j}}
$$

著名的 Beltrami 定理表明一个 Riemann 度量是局部射影平坦的当且仅当它具有 常截面曲率. Finsler 几何中的旗曲率是 Riemann 几何中截面曲率的自然推广. 但是, 流形 $M$ 上每个局部射影平坦的 Finsler 度量 $F$ 具有数量旗曲率, 即旗曲率 $K=K(x, y)$ 是 $T M \backslash\{0\}$ 上的标量函数. 而且, 有许多局部射影平坦的 Finsler 度 量并不具有常旗曲率. 因此, 对于 Finsler 度量, 类似的 Beltrami 定理不再成立.

著名的 Funk 度量 $F=F(x, y)$ 在 $\mathbb{R}^{n}$ 中的强凸域上射影平坦且具有常旗曲率 
$K=-1 / 4$. 当这个域是 $\mathbb{R}^{n}$ 中的单位球 $B^{n}$ 时, Funk 度量由下式给定:

$$
F=\frac{\sqrt{\left(1-|x|^{2}\right)|y|^{2}+\langle x, y\rangle^{2}}}{1-|x|^{2}}+\frac{\langle x, y\rangle}{1-|x|^{2}} .
$$

度量 (1.2) 式具有如下形式:

$$
F=\alpha+\beta,
$$

其中 $\alpha$ 是 Riemann 度量, $\beta=b_{i} y^{i}$ 是 1- 形式. 这种形式的 Finsler 度量称为 Randers 度量. 众所周知, Randers 度量 $F=\alpha+\beta$ 为射影平坦的，当且仅当 $\alpha$ 是射影平 坦的, $\beta$ 是闭的. 沈忠民 ${ }^{[3]}$ 对所有具常旗曲率的射影平坦的 Randers 度量进行了 分类. 最近, 沈忠民和 Yidirim ${ }^{1)}$ 已经研究和分类了有如下形式的局部射影平坦 度量:

$$
F=(\alpha \pm \sqrt{k} \beta)^{2} / \alpha .
$$

他们还确定了具有常旗曲率的这种度量. 这些度量除局部 Minkowski 外, 已经由 莫小欢等 ${ }^{2)}$ 构造. 此外, 杨春红等 ${ }^{[4]}$ 也研究了具有形式

$$
F=\alpha+\varepsilon \beta+k \frac{\beta^{2}}{\alpha}
$$

的局部射影平坦度量.

因此，我们自然要考虑流形上有如下多项式形式的 Finsler 度量:

$$
F=\alpha\left\{1+a_{1} s+a_{2} s^{2}+\cdots+a_{n} s^{n}\right\}, \quad s=\beta / \alpha .
$$

通过简单的分析 (参考 $\S 3$ ), 我们得到这样一类 Finsler 度量:

$$
F=\alpha\left\{1+\varepsilon s+2 k s^{2}-\frac{k^{2}}{3} s^{4}\right\}, \quad s=\frac{\beta}{\alpha},
$$

其中 $\varepsilon$ 和 $k \neq 0$ 为常数. 有 (1.4) 式形式的 Finsler 度量是特殊的 $(\alpha, \beta)$ - 度量 (参考 $\S 2)$. 许多射影平坦的 $(\alpha, \beta)$ - 度量是平凡的, 即 $\alpha$ 是射影平坦的, $\beta$ 关于 $\alpha$ 平行.

本文将对具有 (1.4) 式形式的 Finsler 度量, 找到其为射影平坦的非平凡特 解. 首先, 我们证明了

定理 1.1 Finsler 度量

$$
F=\alpha+\varepsilon \beta+\frac{2 k \beta^{2}}{\alpha}-\frac{k^{2} \beta^{4}}{3 \alpha^{3}}
$$

是射影平坦的，当且仅当

$$
\begin{gathered}
G_{\alpha}^{i}=\eta y^{i}-2 k \tau \alpha^{2} b^{i}, \\
b_{i \mid j}=\tau\left[\left(1+4 k b^{2}\right) a_{i j}-5 k b_{i} b_{j}\right],
\end{gathered}
$$

其中

$$
\tau=\tau(x), \quad \eta=\eta_{i}(x) y^{i},
$$

1) Shen $Z$ M, Yildirim G C. On a class of projectively flat metrics with constant flag curvature

2) Mo $\mathrm{X} \mathrm{H}$, Yang $\mathrm{CH}$. The explicit construction of projectively flat $(\alpha, \beta)$-metrics. Institute of Mathematics, Peking University, 2004-74 
$\varepsilon$ 是常数, $k$ 为非零常数. 此时

$$
G^{i}=(\eta+2 \tau \alpha \chi) y^{i}
$$

其中

$$
\chi:=\frac{\left(1-k s^{2}\right)\left(3 \varepsilon+12 k s-4 k^{2} s^{3}\right)}{4\left(3 \varepsilon s+3+6 k s^{2}-k^{2} s^{4}\right)}-k s, \quad s=\frac{\beta}{\alpha} .
$$

然后, 给出了 (1.5) 和 (1.6) 式的特解.

定理 1.2 设 $\varepsilon$ 是常数, $k$ 为非零常数. 设 $\rho:=\rho(h), h:=h(x)$ 为

$$
\begin{gathered}
\rho=\frac{2}{k} \ln \left(\frac{1}{4} C_{2}^{2} k\left(2 \theta h-\mu h^{2}+2 C_{3}\right)\right), \\
h=\frac{1}{\sqrt{1+\mu|x|^{2}}}\left\{C_{1}+\langle a, x\rangle+\frac{\theta|x|^{2}}{1+\sqrt{1+\mu|x|^{2}}}\right\},
\end{gathered}
$$

其中 $C_{1}, C_{2} \neq 0, C_{3}, \mu$ 和 $\theta$ 是常数, $a \in \mathbb{R}^{n}$ 是常向量. 若取

$$
\alpha:=e^{k \rho} \bar{\alpha}, \quad \beta:=\frac{1}{2} C_{2} e^{3 k \rho / 4} h_{0},
$$

其中

$$
\bar{\alpha}:=\frac{\sqrt{|y|^{2}+\mu\left(|x|^{2}|y|^{2}-\langle x, y\rangle^{2}\right)}}{1+\mu|x|^{2}}, \quad h_{0}=h_{x^{i}} y^{i}
$$

则 Finsler 度量

$$
F=\alpha+\varepsilon \beta+\frac{2 k \beta^{2}}{\alpha}-\frac{k^{2} \beta^{4}}{3 \alpha^{3}}
$$

是射影平坦的.

由定理 1.1 可以确定具常旗曲率的 (1.4) 式形式的 Finsler 度量.

定理 1.3 设

$$
F=\alpha+\varepsilon \beta+\frac{2 k \beta^{2}}{\alpha}-\frac{k^{2} \beta^{4}}{3 \alpha^{3}},
$$

其中 $\varepsilon$ 为常数, 且 $k$ 为非零常数. 如果 $F$ 是具有常旗曲率的局部射影平坦度量, 那么 $\alpha$ 是射影平坦的, 且 $\beta$ 关于 $\alpha$ 平行. 此时, $F$ 为局部 Minkowski 度量.

\section{$2(\alpha, \beta)$ - 度量}

由定义, 一般的 $(\alpha, \beta)$ - 度量可表示为如下形式:

$$
F=\alpha \varphi(s), \quad s=\beta / \alpha,
$$

其中 $\alpha=\sqrt{a_{i j} y^{i} y^{j}}$ 是 Riemann 度量, $\beta=b_{i}(x) y^{i}$ 为 1- 形式. $\varphi=\varphi(s)$ 是开区间 $\left(-b_{0}, b_{0}\right)$ 上的 $C^{\infty}$ 正函数, 满足

$$
\varphi(s)-s \varphi^{\prime}(s)+\left(b^{2}-s^{2}\right) \varphi^{\prime \prime}(s)>0, \quad|s| \leqslant b<b_{0} .
$$

易知 $F$ 是 Finsler 度量当且仅当 $\left\|\beta_{x}\right\|_{\alpha}<b_{0}$ 对任意 $x \in M$ 成立 ${ }^{[5]}$.

记 $b_{i \mid j}$ 为 $\beta$ 关于 $\alpha$ 的共变导数. 令

$$
r_{i j}:=\left(b_{i \mid j}+b_{j \mid i}\right) / 2, \quad s_{i j}:=\left(b_{i \mid j}-b_{j \mid i}\right) / 2,
$$




$$
s_{i}:=b^{j} s_{i j}, \quad s_{j}^{i}:=a^{i k} s_{k j},
$$

及

$$
r_{00}:=r_{i j} y^{i} y^{j}, \quad s_{0}:=s_{i} y^{i}, \quad s_{0}^{i}:=s_{j}^{i} y^{j}, \quad s_{i 0}:=s_{i j} y^{j} .
$$

显然, $\beta$ 是闭的当且仅当 $s_{i j}=0$.

以下分别用 $F_{y^{i}}$ 和 $F_{y^{i} y^{j}}$ 表示 $\frac{\partial F}{\partial y^{i}}$ 和 $\frac{\partial^{2} F}{\partial y^{i} \partial y^{j}}$, 依此类推. 记 $G^{i}$ 和 $G_{\alpha}^{i}$ 分别为 $F$ 和 $\alpha$ 的测地系数, 它们由下式给出:

$$
\begin{aligned}
& G^{i}=g^{i l}\left\{\left[F^{2}\right]_{x^{k} y^{l}} y^{k}-\left[F^{2}\right]_{x^{l}}\right\} / 4, \\
& G_{\alpha}^{i}=a^{i l}\left\{\left[\alpha^{2}\right]_{x^{k} y^{l}} y^{k}-\left[\alpha^{2}\right]_{x^{l}}\right\} / 4,
\end{aligned}
$$

其中

$$
\left(g^{i j}\right):=\left(g_{i j}\right)^{-1}=\left(\left[F^{2}\right]_{y^{i} y^{j}} / 2\right)^{-1}, \quad\left(a^{i j}\right):=\left(a_{i j}\right)^{-1} .
$$

我们有下述引理:

引理 2.1 ${ }^{1)}$ 测地系数 $G^{i}$ 与 $G_{\alpha}^{i}$ 有如下关系:

$$
\begin{aligned}
G^{i}= & G_{\alpha}^{i}+\alpha Q s_{0}^{i}+J\left\{-2 Q \alpha s_{0}+r_{00}\right\} \frac{y^{i}}{\alpha} \\
& +H\left\{-2 Q \alpha s_{0}+r_{00}\right\}\left\{b^{i}-\frac{s y^{i}}{\alpha}\right\},
\end{aligned}
$$

其中

$$
\begin{gathered}
Q:=\frac{\varphi^{\prime}}{\varphi-s \varphi^{\prime}}, \quad J:=\frac{\varphi^{\prime}\left(\varphi-s \varphi^{\prime}\right)}{2 \varphi\left(\left(\varphi-s \varphi^{\prime}\right)+\left(b^{2}-s^{2}\right) \varphi^{\prime \prime}\right)} \\
H:=\frac{\varphi^{\prime \prime}}{2\left(\left(\varphi-s \varphi^{\prime}\right)+\left(b^{2}-s^{2}\right) \varphi^{\prime \prime}\right)}
\end{gathered}
$$

这里

$$
s:=\beta / \alpha, \quad b:=\left\|\beta_{x}\right\|_{\alpha} .
$$

(2.1) 式在文献 [5,6] 中已经给出, 文献 [7,8] 中给出了另一种形式.

由 (1.1) 式有

引理 2.2 ${ }^{1)}$ 一个 $(\alpha, \beta)$ - 度量 $F=\alpha \varphi(s)$ 在开子集 $U \subset \mathbb{R}^{n}$ 上射影平坦，当 且仅当

$$
\left(a_{m l} \alpha^{2}-y_{m} y_{l}\right) G_{\alpha}^{m}+\alpha^{3} Q s_{l 0}+H \alpha\left(-2 \alpha Q s_{0}+r_{00}\right)\left(b_{l} \alpha-s y_{l}\right)=0,
$$

其中 $s=\beta / \alpha$.

\section{3 多项式 $(\boldsymbol{\alpha}, \boldsymbol{\beta})$ - 度量}

本节考虑一种特殊的 $(\alpha, \beta)$ - 度量, 称为多项式 $(\alpha, \beta)$ - 度量. 若 Finsler $(\alpha, \beta)$ 度量具有多项式形式

$$
F:=\alpha\left(1+\sum_{i=1}^{n} a_{i} s^{i}\right)=\alpha\left(1+\sum_{i=1}^{n} \frac{a_{i} \beta^{i}}{\alpha^{i}}\right),
$$

1) 见 249 页脚注 1 
则称为多项式 $(\alpha, \beta)$ - 度量, 其中 $n$ 和 $a_{i}(i=1, \cdots, n)$ 为常数. 若取 $n=1, a_{1}=1$, 则此度量为 Randers 度量; 若取 $n=2, a_{1}=2, a_{2}=1$, 则此度量已由沈忠民和 Yildirim $^{1)}$ 研究; 若取 $n=2, a_{1}=0, a_{2}=1$, 则此度量已由 Sennarath 和 Thornley ${ }^{2)}$ 研究; 若允许 $n$ 趋向于无穷, 并取 $a_{i}=1(i=1,2,3, \cdots)$, 则此度量为 Matsumoto 度量. 本文讨论形如

$$
F=\alpha\left(1+\sum_{i=1}^{4} a_{i} s^{i}\right)
$$

的一类多项式 $(\alpha, \beta)$ - 度量.

由引理 2.1, 可以计算得

$$
\begin{gathered}
Q=\frac{a_{1}+2 a_{2} s+3 a_{3} s^{2}+4 a_{4} s^{3}}{1-a_{2} s^{2}-2 a_{3} s^{3}-3 a_{4} s^{4}}, \\
J=\frac{1}{2} \frac{a_{1}+2 a_{2} s+3 a_{3} s^{2}+4 a_{4} s^{3}}{1+a_{1} s+a_{2} s^{2}+a_{3} s^{3}+a_{4} s^{4}} \\
\cdot \frac{1-a_{2} s^{2}-2 a_{3} s^{3}-3 a_{4} s^{4}}{1-3 a_{2} s^{2}-8 a_{3} s^{3}-15 a_{4} s^{4}+2 b^{2} a_{2}+6 b^{2} a_{3} s+12 b^{2} a_{4} s^{2}}, \\
H=\frac{a_{2}+3 a_{3} s+6 a_{4} s^{2}}{1-3 a_{2} s^{2}-8 a_{3} s^{3}-15 a_{4} s^{4}+2 b^{2} a_{2}+6 b^{2} a_{3} s+12 b^{2} a_{4} s^{2}} .
\end{gathered}
$$

方程 (2.3) 可化为

$$
\begin{gathered}
\left(a_{m l} \alpha^{2}-y_{m} y_{l}\right) G_{\alpha}^{m}+\frac{\left(a_{1}+2 a_{2} s+3 a_{3} s^{2}+4 a_{4} s^{3}\right) \alpha^{3} s_{l 0}}{1-a_{2} s^{2}-2 a_{3} s^{3}-3 a_{4} s^{4}} \\
+\frac{\left(a_{2}+3 a_{3} s+6 a_{4} s^{2}\right)}{1-3 a_{2} s^{2}-8 a_{3} s^{3}-15 a_{4} s^{4}+2 b^{2} a_{2}+6 b^{2} a_{3} s+12 b^{2} a_{4} s^{2}} \\
\cdot\left\{-2 \alpha \frac{a_{1}+2 a_{2} s+3 a_{3} s^{2}+4 a_{4} s^{3}}{1-a_{2} s^{2}-2 a_{3} s^{3}-3 a_{4} s^{4}} s_{0}+r_{00}\right\}\left(b_{l} \alpha^{2}-\beta y_{l}\right)=0 .
\end{gathered}
$$

我们希望当形如 (3.1) 式的度量为射影平坦时, 1- 形式 $\beta$ 是闭的. 此时 (3.2) 式可简化为

$$
+\frac{\left(a_{m l} \alpha^{2}-y_{m} y_{l}\right) G_{\alpha}^{m}}{1-3 a_{2} s^{2}-8 a_{3} s^{3}-15 a_{4} s^{4}+2 b^{2} a_{2}+6 b^{2} a_{3} s+12 b^{2} a_{4} s^{2}}=0 .
$$

因此假设 $r_{00}$ 取如下形式:

$$
r_{00}=p \alpha^{2}+q \beta^{2},
$$

使得 (3.3) 式左边分式部分变成形如 $\tilde{p} \alpha^{2}+\tilde{q} \beta^{2}$ 的多项式. 我们有

$$
\frac{\left(a_{2}+3 a_{3} s+6 a_{4} s^{2}\right)\left(p+q s^{2}\right)}{1-3 a_{2} s^{2}-8 a_{3} s^{3}-15 a_{4} s^{4}+2 b^{2} a_{2}+6 b^{2} a_{3} s+12 b^{2} a_{4} s^{2}}=\tilde{p}+\tilde{q} s^{2},
$$

其中 $p, q, \tilde{p}$ 和 $\tilde{q}$ 是依赖于 $b:=\|\beta\|_{\alpha}$ 的函数. 通过简单的分析, 可见当

$$
a_{3}=0, \quad a_{2}=2 k, \quad a_{4}=-\frac{1}{3} k^{2}, \quad k=\mathrm{const} \neq 0
$$

1) 见 249 页脚注 1

2) Senarath P, Thornley G M. Locally projectively flat Finsler spaces with $(\alpha, \beta)$-metrics. 2004 
时, 这是可能的. 所以我们考虑形如 (1.4) 式的 Finsler 度量.

$$
4 F=\alpha+\varepsilon \beta+\frac{2 k \beta^{2}}{\alpha}-\frac{k^{2} \beta^{4}}{3 \alpha^{3}}
$$

本节考虑有如下形式的 $(\alpha, \beta)$ - 度量:

$$
F=\alpha+\varepsilon \beta+\frac{2 k \beta^{2}}{\alpha}-\frac{k^{2} \beta^{4}}{3 \alpha^{3}},
$$

其中 $k \neq 0, \varepsilon$ 是常数, $\alpha=\sqrt{a_{i j} y^{i} y^{j}}$ 是 Riemann 度量, $\beta=b_{i} y^{i}$ 是流形 $M$ 上的 1形式.

引理 4.1 形如 (4.1) 式的度量 $F$ 是一个 Finsler 度量, 当且仅当

$$
b:=\left\|\beta_{x}\right\|_{\alpha}<1 / \sqrt{|k|}, \quad \forall x \in M \text {. }
$$

证 记

$$
\varphi=1+\varepsilon s+2 k s^{2}-\frac{k^{2} s^{4}}{3}, \quad s=\frac{\beta}{\alpha} .
$$

由 $(\alpha, \beta)$ - 度量的定义, 需要保证

$$
\varphi-s \varphi^{\prime}+\left(b^{2}-s^{2}\right) \varphi^{\prime \prime}=\left(k s^{2}-1\right)\left(5 k s^{2}-1-4 k b^{2}\right)>0 .
$$

对任意的 $|s| \leqslant b<1 / \sqrt{|k|}$, 有

$$
\varphi-s \varphi^{\prime}+\left(b^{2}-s^{2}\right) \varphi^{\prime \prime} \geqslant\left(k b^{2}-1\right)^{2}>0 .
$$

证毕.

从现在起总是假定 $\varepsilon$ 满足

$$
1+\varepsilon s+2 k s^{2}-\frac{k^{2} s^{4}}{3}>0, \quad \forall|s| \leqslant b<\frac{1}{\sqrt{|k|}} .
$$

由引理 2.1 可以计算得

$$
\begin{gathered}
Q=\frac{3 \varepsilon+12 k s-4 k^{2} s^{3}}{3\left(1-2 k s^{2}+k^{2} s^{4}\right)}, \\
J=\frac{\left(1-k s^{2}\right)\left(3 \varepsilon+12 k s-4 k^{2} s^{3}\right)}{2\left(1+4 k b^{2}-5 k s^{2}\right)\left(3 \varepsilon s+3+6 k s^{2}-k^{2} s^{4}\right)}, \\
H=\frac{2 k}{1+4 k b^{2}-5 k s^{2}} .
\end{gathered}
$$

方程 (2.3) 化为

$$
\begin{aligned}
0= & \left(a_{m l} \alpha^{2}-y_{m} y_{l}\right) G_{\alpha}^{m}+\frac{\left(3 \varepsilon+12 k s-4 k^{2} s^{3}\right) \alpha^{3} s_{l 0}}{3\left(1-k s^{2}\right)^{2}} \\
& +\frac{2 k \alpha}{\left(4 k b^{2}+1\right)-5 k s^{2}}\left\{-2 \alpha \frac{3 \varepsilon+12 k s-4 k^{2} s^{3}}{3\left(1-k s^{2}\right)^{2}} s_{0}+r_{00}\right\}\left(b_{i} \alpha-s y_{l}\right) .
\end{aligned}
$$

利用 (4.4) 式可以证明定理 1.1.

定理 1.1 的证 首先, 将 (4.4) 式改写为关于 $y^{i}$ 和 $\alpha$ 的多项式

$$
\begin{aligned}
0= & 3\left(\alpha^{2}-k \beta^{2}\right)^{2}\left[\left(4 k b^{2}+1\right) \alpha^{2}-5 k \beta^{2}\right]\left(a_{m l} \alpha^{2}-y_{m} y_{l}\right) G_{\alpha}^{m} \\
& +\alpha^{4}\left(3 \varepsilon \alpha^{3}+12 k \beta \alpha^{2}-4 k^{2} \beta^{3}\right) s_{l 0}\left[\left(4 k b^{2}+1\right) \alpha^{2}-5 k \beta^{2}\right] \\
& +2 k \alpha^{2}\left[-2 \alpha^{2}\left(3 \varepsilon \alpha^{3}+12 k \beta \alpha^{2}-4 k^{2} \beta^{3}\right) s_{0}+3 r_{00}\left(\alpha^{2}-k \beta^{2}\right)^{2}\right]\left(b_{l} \alpha^{2}-\beta y_{l}\right) .
\end{aligned}
$$


$\alpha$ 的系数必为零 (注意, $\alpha$ 的偶次方是关于 $y^{i}$ 的多项式). 我们得到

$$
3 \varepsilon \alpha^{7} s_{l 0}\left[\left(4 k b^{2}+1\right) \alpha^{2}-5 k \beta^{2}\right]=12 k \varepsilon \alpha^{7} s_{0}\left(b_{l} \alpha^{2}-\beta y_{l}\right) .
$$

设 $\varepsilon \neq 0$, 则

$$
s_{l 0}\left[\left(4 k b^{2}+1\right) \alpha^{2}-5 k \beta^{2}\right]=4 k s_{0}\left(b_{l} \alpha^{2}-\beta y_{l}\right) .
$$

对 (4.6) 式用 $b^{l}$ 缩并, 得到

$$
\left(\alpha^{2}-k \beta^{2}\right) s_{0}=0
$$

由于 $\alpha^{2}-k \beta^{2} \neq 0$, 故有

$$
s_{0}=0 .
$$

由(4.6) 式, 有

$$
s_{l 0}=0 .
$$

所以, $\beta$ 是闭的.

设 $\varepsilon=0$, 则 (4.5) 式化为

$$
\begin{aligned}
0= & 3\left(\alpha^{2}-k \beta^{2}\right)^{2}\left[\left(4 k b^{2}+1\right) \alpha^{2}-5 k \beta^{2}\right]\left(a_{m l} \alpha^{2}-y_{m} y_{l}\right) G_{\alpha}^{m} \\
& +4 k \alpha^{4}\left(3 \beta \alpha^{2}-k \beta^{3}\right) s_{l 0}\left[\left(4 k b^{2}+1\right) \alpha^{2}-5 k \beta^{2}\right] \\
& +2 k \alpha^{2}\left[-8 k \alpha^{2}\left(3 \beta \alpha^{2}-k \beta^{3}\right) s_{0}+3 r_{00}\left(\alpha^{2}-k \beta^{2}\right)^{2}\right]\left(b_{l} \alpha^{2}-\beta y_{l}\right) .
\end{aligned}
$$

改写上式, 得

$$
\begin{aligned}
& 3\left(\alpha^{2}-k \beta^{2}\right)^{2}\left\{\left[\left(4 k b^{2}+1\right) \alpha^{2}-5 k \beta^{2}\right]\left(a_{m l} \alpha^{2}-y_{m} y_{l}\right) G_{\alpha}^{m}+2 k r_{00} \alpha^{2}\left(b_{l} \alpha^{2}-y_{l} \beta\right)\right\} \\
= & -4 k \alpha^{4} \beta\left(3 \alpha^{2}-k \beta^{2}\right)\left\{s_{l 0}\left[\left(4 k b^{2}+1\right) \alpha^{2}-5 k \beta^{2}\right]-4 k s_{0}\left(b_{l} \alpha^{2}-\beta y_{l}\right)\right\} .
\end{aligned}
$$

因为 $k \alpha^{4} \beta\left(3 \alpha^{2}-k \beta^{2}\right)$ 不能被 $\left(\alpha^{2}-k \beta^{2}\right)^{2}$ 整除, 所以 $s_{l 0}\left[\left(4 k b^{2}+1\right) \alpha^{2}-5 k \beta^{2}\right]-$ $4 k s_{0}\left(b_{l} \alpha^{2}-\beta y_{l}\right)$ 必被 $\left(\alpha^{2}-k \beta^{2}\right)^{2}$ 整除. 这是不可能的, 除非

$$
s_{l 0}\left[\left(4 k b^{2}+1\right) \alpha^{2}-5 k \beta^{2}\right]-4 k s_{0}\left(b_{l} \alpha^{2}-\beta y_{l}\right)=0 .
$$

类似于上面的讨论, 可得 $\beta$ 是闭的.

因为 $s_{i j}=0$, 方程 (4.5) 可化为

$$
\left[\left(4 k b^{2}+1\right) \alpha^{2}-5 k \beta^{2}\right]\left(a_{m l} \alpha^{2}-y_{m} y_{l}\right) G_{\alpha}^{m}+2 k \alpha^{2} r_{00}\left(b_{l} \alpha^{2}-\beta y_{l}\right)=0 .
$$

对 (4.9) 式用 $b^{l}$ 缩并, 得到

$$
\left[\left(4 k b^{2}+1\right) \alpha^{2}-5 k \beta^{2}\right]\left(b_{m} \alpha^{2}-y_{m} \beta\right) G_{\alpha}^{m}=-2 k \alpha^{2} r_{00}\left(b^{2} \alpha^{2}-\beta^{2}\right) .
$$

注意到多项式 $\left(4 k b^{2}+1\right) \alpha^{2}-5 k \beta^{2}$ 不能被 $\alpha^{2}$ 和 $b^{2} \alpha^{2}-\beta^{2}$ 整除, 因此 $\left(b_{m} \alpha^{2}-\right.$ $\left.y_{m} \beta\right) G_{\alpha}^{m}$ 被 $k \alpha^{2}\left(b^{2} \alpha^{2}-\beta^{2}\right)$ 整除. 所以, 存在标量函数 $\tau=\tau(x)$, 使得

$$
r_{00}=\tau\left[\left(4 k b^{2}+1\right) \alpha^{2}-5 k \beta^{2}\right] .
$$

由 (4.8) 和 (4.11) 式, 关于 $G^{i}$ 的 (2.1) 式可简化为

$$
G^{i}=G_{\alpha}^{i}+2 \tau \chi \alpha y^{i}+2 k \tau \alpha^{2} b^{i},
$$


其中 $\chi$ 由 (1.8) 式给出.

下面计算 $G_{\alpha}^{i}$. 将 (4.11) 式代入 (4.9) 式并用 $b^{l}$ 缩并, 得到

$$
\left(b_{m} \alpha^{2}-\beta y_{m}\right) G_{\alpha}^{m}+2 k \tau \alpha^{2}\left(b^{2} \alpha^{2}-\beta^{2}\right)=0 .
$$

改写 (4.13) 式为

$$
\alpha^{2}\left(G_{\alpha}^{m} b_{m}+2 k \tau b^{2} \alpha^{2}\right)=\beta\left(y_{m} G_{\alpha}^{m}+2 k \tau \alpha^{2} \beta\right) .
$$

因为 $\alpha^{2}$ 不能被 $\beta$ 整除, 所以存在 1 - 形式 $\eta:=\eta_{i} y^{i}$, 使得

$$
\begin{aligned}
& G_{\alpha}^{m} b_{m}+2 k \tau b^{2} \alpha^{2}=\eta \beta, \\
& G_{\alpha}^{m} y_{m}+2 k \tau \alpha^{2} \beta=\eta \alpha^{2} .
\end{aligned}
$$

由上述两方程的任意一个, 都有

$$
G_{\alpha}^{i}=\eta y^{i}-2 k \tau \alpha^{2} b^{i} .
$$

这就证明了 (1.5) 式. 由于

$$
d \beta=0, \quad r_{00}=\tau\left[\left(4 k b^{2}+1\right) \alpha^{2}-5 k \beta^{2}\right],
$$

可得 (1.6) 式. 此时, $G^{i}$ 由 (1.7) 式给定. 证毕.

\section{5 旗曲率}

本节将考虑具有常旗曲率 $K=\lambda$ 的 Finsler 度量

$$
F=\alpha+\varepsilon \beta+\frac{2 k \beta^{2}}{\alpha}-\frac{k^{2} \beta^{4}}{3 \alpha^{3}},
$$

其中 $\varepsilon$ 为常数, $k$ 为非零常数. 假定 $F$ 是局部射影平坦的, 使得在局部坐标系 下, $F$ 的测地系数是 (1.7) 式. 我们知道, 如果 $F$ 的测地系数具有形式 $G^{i}=P y^{i}$, 那么 $F$ 有标量旗曲率

$$
K=\frac{P^{2}-P_{x^{k}} y^{k}}{F^{2}} .
$$

因此

$$
K=\frac{(\eta+2 \tau \chi \alpha)^{2}-\eta_{x^{k}} y^{k}-2 \tau_{x^{k}} y^{k} \alpha \chi-2 \tau \chi^{\prime}(s) s_{x^{k}} y^{k} \alpha-2 \tau \chi \alpha_{x^{k}} y^{k}}{F^{2}} .
$$

注意到

$$
\begin{gathered}
s_{x^{k}} y^{k}=\frac{r_{00}}{\alpha}+\frac{2}{\alpha^{2}}\left(b_{m} \alpha-s y_{m}\right) G_{\alpha}^{m}=\tau \alpha\left(1-k s^{2}\right), \\
\alpha_{x^{k}} y^{k}=\frac{2}{\alpha} G_{\alpha}^{m} y_{m}=2(\eta-2 k \tau \beta) \alpha,
\end{gathered}
$$

我们有

$$
K=\frac{\eta^{2}-\eta_{x^{k}} y^{k}+4 \tau^{2} \chi^{2} \alpha^{2}-2 \tau_{x^{k}} y^{k} \chi \alpha-2 \tau^{2} \alpha^{2} \chi^{\prime}\left(1-k s^{2}\right)+8 k \tau^{2} s \chi \alpha^{2}}{F^{2}} .
$$

引理 5.1 如果

$$
F=\alpha+\varepsilon \beta+\frac{2 k \beta^{2}}{\alpha}-\frac{k^{2} \beta^{4}}{3 \alpha^{3}}
$$


是射影平坦的, 且具有常旗曲率 $K=\lambda=$ 常数, 其中 $\varepsilon$ 是常数, $k$ 是非零常数, 那么 $\lambda=0$.

证 首先, 由 (5.2) 式, 等式 $K=\lambda$ 乘以 $81 \alpha^{12} F^{4}$, 可以得到

$$
A \alpha^{5}+B \alpha^{4}+12 k^{6} \varepsilon \lambda \beta^{13} \alpha^{3}+24 k^{7} \lambda \beta^{14} \alpha^{2}-k^{8} \lambda \beta^{16}=0,
$$

其中 $A$ 和 $B$ 分别是关于 $y$ 的齐 11 次和齐 12 次多项式. 将上式改写为

$$
\left(A \alpha^{2}+12 k^{6} \lambda \varepsilon \beta^{13}\right) \alpha^{3}+\left(B \alpha^{4}+24 k^{7} \lambda \beta^{14} \alpha^{2}-k^{8} \lambda \beta^{16}\right)=0,
$$

则有

$$
A \alpha^{2}+12 k^{6} \lambda \varepsilon \beta^{13}=0, \quad\left(B \alpha^{2}+24 k^{7} \lambda \beta^{14}\right) \alpha^{2}=k^{8} \lambda \beta^{16} .
$$

由于 $\beta^{2}$ 不能被 $\alpha$ 和 $k \neq 0$ 整除，从 (5.3) 式的第 2 式就有 $\lambda=0$. 证毕.

考虑 (1.6) 式中 $\tau=0$ 的情形. 此时

$$
b_{i \mid j}=0, \quad G^{i}=G_{\alpha}^{i}=\eta y^{i} .
$$

由引理 $5.1, F$ 有零旗曲率, 因此 $\alpha$ 有零截面曲率, 即 $\alpha$ 局部与 Euclid 度量等距. 所以有

\section{命题 5.2 设}

$$
F=\alpha+\varepsilon \beta+\frac{2 k \beta^{2}}{\alpha}-\frac{k^{2} \beta^{4}}{3 \alpha^{3}}
$$

其中 $\varepsilon$ 是常数, $k$ 为非零常数. 假定 $F$ 是具有零旗曲率的局部射影平坦度量. 如 果 $\tau=0$, 那么 $\alpha$ 是平坦度量, 且 $\beta$ 关于 $\alpha$ 平行. 此时, $F$ 是局部 Minkowski 度 量.

由引理 5.1 和命题 5.2, 可以证明定理 1.3.

定理 1.3 的证 只需证明 $\tau \equiv 0$.

在 $K=0$ 的假设下，从 (5.2) 式有

$$
\Phi \alpha^{3}+\Psi=0,
$$

其中

$$
\begin{aligned}
\Phi:=( & \left.324 k \tau^{2} \varepsilon \beta-18 \tau_{0} \varepsilon\right) \alpha^{6}+\left(72\left(\eta^{2}-\eta_{0}\right) \varepsilon \beta-252 k^{2} \tau^{2} \varepsilon \beta^{3}+54 k \tau_{0} \varepsilon \beta^{2}\right) \alpha^{4} \\
+ & \left(144 k\left(\eta^{2}-\eta_{0}\right) \varepsilon \beta^{3}-1044 k^{3} \tau^{2} \varepsilon \beta^{5}+426 k^{2} \tau_{0} \varepsilon \beta^{4}\right) \alpha^{2} \\
+ & \left(-24 k^{2}\left(\eta^{2}-\eta_{0}\right) \varepsilon+204 k^{4} \tau^{2} \varepsilon \beta^{2}-78 k^{3} \tau_{0} \varepsilon \beta\right) \beta^{5} \\
\Psi:= & 27 \tau^{2} \varepsilon^{2} \alpha^{10}+\left(36\left(\eta^{2}-\eta_{0}\right)+\left(54 k \varepsilon^{2}+720 k^{2}\right) \beta^{2} \tau^{2}-18 \tau_{0} \varepsilon^{2} \beta\right) \alpha^{8} \\
& +\left(\left(36 \varepsilon^{2}+144 k\right)\left(\eta^{2}-\eta_{0}\right) \beta^{2}-\left(225 k^{2} \varepsilon^{2}+1440 k^{3}\right) \beta^{4} \tau^{2}\right. \\
& \left.+\left(90 k \varepsilon^{2}+240 k^{2}\right) \tau_{0} \beta^{3}\right) \alpha^{6} \\
& +\left(120 k^{2}\left(\eta^{2}-\eta_{0}\right) \beta^{4}-576 k^{4} \tau^{2} \beta^{6}+432 k^{3} \tau_{0} \beta^{5}\right) \alpha^{4} \\
& +\left(-48 k^{3}\left(\eta^{2}-\eta_{0}\right) \beta^{6}+288 k^{5} \tau^{2} \beta^{8}-176 k^{4} \tau_{0} \beta^{7}\right) \alpha^{2} \\
& +\left(4 k^{4}\left(\eta^{2}-\eta_{0}\right)-16 k^{6} \tau^{2} \beta^{2}+16 k^{5} \tau_{0} \beta\right) \beta^{8}
\end{aligned}
$$


其中

$$
\eta_{0}:=\eta_{x^{k}} y^{k}, \quad \tau_{0}:=\tau_{x^{k}} y^{k} .
$$

注意到 $\Phi$ 和 $\Psi$ 都是关于 $y$ 的齐次多项式, $\alpha=\sqrt{a_{i j} y^{i} y^{j}}$ 是根式, (5.4) 式表明

$$
\Phi=0, \quad \Psi=0 .
$$

下面分两种情形讨论:

情形 $1 \quad \varepsilon=0$. 此时, $\Phi \equiv 0$, 只需考虑 $\Psi=0$, 有

$$
\begin{aligned}
0= & \left(36\left(\eta^{2}-\eta_{0}\right)+720 k^{2} \tau^{2} \beta^{2}\right) \alpha^{8} \\
& +\left(144 k\left(\eta^{2}-\eta_{0}\right) \beta^{2}-1440 k^{3} \tau^{2} \beta^{4}+240 k^{2} \tau_{0} \beta^{3}\right) \alpha^{6} \\
& +\left(120 k^{2}\left(\eta^{2}-\eta_{0}\right) \beta^{4}-576 k^{4} \tau^{2} \beta^{6}+432 k^{3} \tau_{0} \beta^{5}\right) \alpha^{4} \\
& +\left(-48 k^{3}\left(\eta^{2}-\eta_{0}\right) \beta^{6}+288 k^{5} \tau^{2} \beta^{8}-176 k^{4} \tau_{0} \beta^{7}\right) \alpha^{2} \\
& +\left(4 k^{4}\left(\eta^{2}-\eta_{0}\right)-16 k^{6} \tau^{2} \beta^{2}+16 k^{5} \tau_{0} \beta\right) \beta^{8} .
\end{aligned}
$$

因为 $\beta^{2}$ 不能被 $\alpha$ 整除, 所以存在标量函数 $c_{i}=c_{i}(x)(i=1,2,3,4)$, 使得

$$
\begin{gathered}
4 k^{4}\left(\eta^{2}-\eta_{0}\right)-18 k^{6} \tau^{2} \beta^{2}+16 k^{5} \tau_{0} \beta=c_{1} \alpha^{2}, \\
c_{1} \beta^{2}-48 k^{3}\left(\eta^{2}-\eta_{0}\right)+288 k^{5} \tau^{2} \beta^{2}-176 k^{4} \tau_{0} \beta=c_{2} \alpha^{2}, \\
c_{2} \beta^{2}+120 k^{2}\left(\eta^{2}-\eta_{0}\right)-576 k^{4} \tau^{2} \beta^{2}+432 k^{3} \tau_{0} \beta=c_{3} \alpha^{2}, \\
c_{3} \beta^{2}+144 k\left(\eta^{2}-\eta_{0}\right)-1440 k^{3} \tau^{2} \beta^{2}+240 k^{2} \tau_{0} \beta=c_{4} \alpha^{2},
\end{gathered}
$$

则 (5.6) 式化为

$$
36\left(\eta^{2}-\eta_{0}\right)+720 k^{2} \tau^{2} \beta^{2}=-c_{4} \beta^{2} .
$$

由上式知道 $\eta^{2}-\eta_{0}$ 必包含因子 $\beta^{2}$, 所以存在标量函数 $c_{5}=c_{5}(x)$, 使得

$$
\eta^{2}-\eta_{0}=c_{5} \beta^{2} \text {. }
$$

因此, 由 (5.11) 式可得

$$
\tau^{2}=-\frac{36 c_{5}+c_{4}}{720 k^{2}}
$$

将 (5.12) 和 (5.13) 式代入 (5.10) 式, 有

$$
\left(c_{3} \beta+216 k c_{5} \beta+2 k c_{4} \beta+240 k^{2} \tau_{0}\right) \beta=c_{4} \alpha^{2} .
$$

由于 $\beta^{2}$ 不能被 $\alpha$ 整除, 故有

$$
c_{4}=0, \quad \tau_{0}=-\frac{216 k c_{5}+c_{3}}{240 k^{2}} \beta, \quad \tau^{2}=-\frac{c_{5}}{20 k^{2}} .
$$

将 (5.12) 和 (5.15) 式代入 (5.9) 式, 可得

$$
\left(c_{2}-240 k^{2} c_{5}-\frac{9 k}{5} c_{3}\right) \beta^{2}=c_{3} \alpha^{2},
$$

故有

$$
c_{3}=0, \quad c_{2}=240 k^{2} c_{5}
$$


将 (5.12)、(5.15) 和 (5.17) 式代入 (5.8) 式, 有

$$
\left(c_{1}+96 k^{3} c_{5}\right) \beta^{2}=240 k^{2} c_{5} \alpha^{2} .
$$

因为 $k \neq 0$, 所以

$$
c_{5}=0, \quad c_{1}=0 .
$$

把 (5.19) 式代入 (5.15) 式的第 3 式, 有 $\tau^{2}=0$, 从而 $\tau=0$.

情形 $2 \varepsilon \neq 0$. 从 $\Phi=0$ 开始计算, 即

$$
\begin{aligned}
& \left\{\left(54 k \tau^{2} \beta-3 \tau_{0}\right) \alpha^{4}+\left[12\left(\eta^{2}-\eta_{0}\right) \beta-42 k^{2} \tau^{2} \beta^{3}+9 k \tau_{0} \beta^{2}\right] \alpha^{2}\right. \\
& \left.+\left[24 k\left(\eta^{2}-\eta_{0}\right) \beta^{3}-174 k^{3} \tau^{2} \beta^{5}+71 k^{2} \tau_{0} \beta^{4}\right]\right\} \alpha^{2} \\
= & -\left[-4 k^{2}\left(\eta^{2}-\eta_{0}\right)+34 k^{4} \tau^{2} \beta^{2}-13 k^{3} \tau_{0} \beta\right] \beta^{5} .
\end{aligned}
$$

由于 $\beta^{2}$ 不能被 $\alpha$ 整除, 故存在标量函数 $d_{i}=d_{i}(x)(i=1,2,3)$, 使得

$$
\begin{gathered}
-4 k^{4}\left(\eta^{2}-\eta_{0}\right)+34 k^{4} \tau^{2} \beta^{2}-13 k^{3} \tau_{0} \beta=d_{1} \alpha^{2}, \\
d_{1} \beta^{2}+24 k\left(\eta^{2}-\eta_{0}\right)-174 k^{3} \tau^{2} \beta^{2}+71 k^{2} \tau_{0} \beta=d_{2} \alpha^{2}, \\
d_{2} \beta^{2}+12\left(\eta^{2}-\eta_{0}\right)-42 k^{2} \tau^{2} \beta^{2}+9 k \tau_{0} \beta=d_{3} \alpha^{2},
\end{gathered}
$$

故 (5.20) 式变为

$$
54 k \tau^{2} \beta-3 \tau_{0}=-\beta d_{3} .
$$

由 (5.24) 式可知 $\tau_{0}$ 必包含因子 $\beta$, 所以必存在标量函数 $d_{4}=d_{4}(x)$, 使得

$$
\tau_{0}=d_{4} \beta \text {. }
$$

代入 (5.24) 式, 可得

$$
\tau^{2}=\frac{3 d_{4}-d_{3}}{54 k}
$$

将 (5.25) 和 (5.26) 式代入 (5.22) 和 (5.23) 式, 有

$$
\begin{gathered}
d_{1} \beta^{2}+24 k\left(\eta^{2}-\eta_{0}\right)-\frac{29 k^{2}}{9}\left(3 d_{4}-d_{3}\right) \beta^{2}+71 k^{2} d_{4} \beta^{2}=d_{2} \alpha^{2}, \\
d_{2} \beta^{2}+12\left(\eta^{2}-\eta_{0}\right)-\frac{7 k}{9}\left(3 d_{4}-d_{3}\right) \beta^{2}+9 k d_{4} \beta^{2}=d_{3} \alpha^{2} .
\end{gathered}
$$

(5.28) 式乘以 $-2 k$ 后加到 (5.27) 式上, 可得

$$
\left(d_{1}-2 k d_{2}+\frac{5 k^{2}}{3} d_{3}+48 k^{2} d_{4}\right) \beta^{2}=\left(d_{2}-2 k d_{3}\right) \alpha^{2} .
$$

因为 $\alpha^{2}$ 不能被 $\beta$ 整除, 故有

$$
d_{2}=2 k d_{3}, \quad d_{1}=\frac{7 k^{2}}{3} d_{3}-48 k^{2} d_{4} .
$$

将 (5.25)、(5.26) 和 (5.30) 式代入 (5.28) 式, 有

$$
\eta^{2}-\eta_{0}=\frac{1}{12}\left\{d_{3} \alpha^{2}-\frac{25 k}{9} d_{3} \beta^{2}-\frac{20 k}{3} d_{4} \beta^{2}\right\} .
$$


将 (5.25)、(5.26)、(5.30) 和 (5.31) 式代入 (5.20) 式, 可得

$$
\left(\frac{8}{3} d_{3}-48 d_{4}\right) \alpha^{2}=\left(\frac{8}{27} d_{3}-\frac{80}{9} d_{4}\right) k \beta^{2} .
$$

由于 $\beta^{2}$ 不能被 $\alpha$ 和 $k \neq 0$ 整除, 所以有

$$
\frac{8}{3} d_{3}-48 d_{4}=0, \quad \frac{8}{27} d_{3}-\frac{80}{9} d_{4}=0 .
$$

解上述方程组, 得

$$
d_{3}=d_{4}=0
$$

从而

$$
\tau^{2}=\frac{3 d_{4}-d_{3}}{54 k}=0
$$

即 $\tau=0$. 证毕.

\section{6 (1.5) 和 (1.6) 的特解}

本节将给出 (1.5) 和 (1.6) 式的特解, 使得 $\tau \beta$ 是闭的. 方法与莫小欢、杨春 红等 ${ }^{[4] 1)}$ 和沈忠民 ${ }^{2)}$ 的方法类似.

由于 $\tau=0$ 是平凡的, 故考虑 $\tau \neq 0$ 的情形.

设 $\tau \beta$ 是闭的, 即局部地, 存在标量函数 $\rho=\rho(x)$, 使得

$$
\tau b_{i}=\rho_{x^{i}} / 4 \text {. }
$$

因为由 (1.6) 式知 $\beta$ 是闭的, 故可设 $\beta=\sigma_{0} / 4$, 其中 $\sigma_{0}:=\sigma_{x^{i}} y^{i}, \sigma=\sigma(x)$ 是标量 函数.

我们有

$$
4 \tau \beta=\tau \sigma_{0}=\rho_{0},
$$

其中 $\rho_{0}:=\rho_{x^{i}} y^{i}$.

设 $\alpha:=e^{k \rho} \bar{\alpha}$, 其中

$$
\bar{\alpha}:=\frac{\sqrt{|y|^{2}+\mu\left(|x|^{2}|y|^{2}-\langle x, y\rangle^{2}\right)}}{1+\mu|x|^{2}} .
$$

$\alpha$ 的测地系数由下式给定:

$$
\begin{aligned}
G_{\alpha}^{i} & =G_{\bar{\alpha}}^{i}+k \rho_{0} y^{i}-\frac{1}{2} k \bar{a}^{i j} \rho_{x^{j}} \bar{\alpha}^{2} \\
& =\left\{-\frac{\mu\langle x, y\rangle}{1+\mu\left|x^{2}\right|}+k \rho_{0}\right\} y^{i}-\frac{1}{2} k a^{i j} \rho_{x^{j}} \alpha^{2} .
\end{aligned}
$$

因此 (1.5) 式满足.

1) 见 249 页脚注 2

2) Shen $Z$ M. On some projectively flat Finsler metrics 
下面采用沈忠民 ${ }^{1)}$ 的方法解 (1.6) 式. 因为 $\beta$ 是闭的, 故 $r_{00}=b_{0 \mid 0}$. 由 (6.2) 和 (6.3) 式, 有

$$
\begin{aligned}
r_{00} & =\frac{\partial b_{i}}{\partial x^{j}} y^{i} y^{j}-2 b_{i} G_{\alpha}^{i} \\
& =\frac{1}{4} \sigma_{00}-\frac{\sigma_{0}}{2}\left\{-\frac{\mu\langle x, y\rangle}{1+\mu|x|^{2}}+k \tau \sigma_{0}\right\}+4 k \tau b^{2} \alpha^{2} .
\end{aligned}
$$

(6.4) 式等价于

$$
\sigma_{00}+\frac{2 \mu\langle x, y\rangle}{1+\mu|x|^{2}} \sigma_{0}=4 \tau \alpha^{2}+\frac{3 k \tau \sigma_{0}^{2}}{4} .
$$

假设 $\rho=\rho(x)$ 和 $\sigma=\sigma(x)$ 由同一个函数 $h=h(x)$ 按如下形式决定:

$$
\rho=\rho(h), \quad \sigma=\sigma(h),
$$

则 (6.2) 式等价于

$$
\tau \sigma^{\prime}=\rho^{\prime} .
$$

(6.5) 式化为

$$
h_{00}+\frac{2 \mu\langle x, y\rangle}{1+\mu|x|^{2}} h_{0}=\frac{4 \rho^{\prime}}{\left(\sigma^{\prime}\right)^{2}} e^{2 k \rho} \bar{\alpha}^{2}+\left(\frac{3 k \rho^{\prime}}{4}-\frac{\sigma^{\prime \prime}}{\sigma^{\prime}}\right) h_{0}^{2} .
$$

引理 6.1 ${ }^{1)}$ 设

$$
h=\frac{1}{\sqrt{1+\mu|x|^{2}}}\left\{C_{1}+\langle a, x\rangle+\frac{\theta|x|^{2}}{1+\sqrt{1+\mu|x|^{2}}}\right\},
$$

其中 $\theta$ 和 $C_{1}$ 是任意常数, $a \in \mathbb{R}^{n}$ 是常向量, 则 $h$ 满足

$$
h_{00}+\frac{2 \mu\langle x, y\rangle}{1+\mu|x|^{2}} h_{0}=(\theta-\mu h) \bar{\alpha}^{2} .
$$

比较 (6.6) 和 (6.7) 式看到, 如果 $\sigma$ 满足

$$
\begin{gathered}
\frac{3 k \rho^{\prime}}{4}-\frac{\sigma^{\prime \prime}}{\sigma^{\prime}}=0, \\
\frac{4 \rho^{\prime}}{\left(\sigma^{\prime}\right)^{2}} e^{2 k \rho}=\theta-\mu h,
\end{gathered}
$$

则 (6.6) 式成立.

由 (6.8) 式, 有

$$
\sigma^{\prime}=2 C_{2} e^{3 k \rho / 4},
$$

其中 $C_{2}$ 是正常数. 将 (6.10) 式代入 (6.9) 式, 可得

$$
\rho^{\prime} e^{k \rho / 2}=C_{2}^{2}(\theta-\mu h) .
$$

解此方程得

$$
\rho=\frac{2}{k} \ln \left(\frac{1}{4} C_{2}^{2} k\left(2 \theta h-\mu h^{2}+2 C_{3}\right)\right),
$$

其中 $C_{3}$ 为常数, 从而定理得证.

1) 见 259 页脚注 2 
致谢 感谢沈忠民教授提供有益的意见和帮助.

\section{参考文献}

1 Hilbert D. Mathematical Problems. Bull Amer Math Soc, 2001, 37: 407 436; Reprinted from Bull Amer Math Soc, 1902, 8: 437 479

2 Hamel G. Über die Geometrieen in denen die Geraden die Kürzesten sind. Math Ann, 1903, 57: 231 264

3 Shen Z. Projectively flat Randers metrics of constant flag curvature. Math Ann, 2003, 325: 19 30

4 杨春红, 莫小欢, 沈忠民. 一些射影平坦 Finsler 度量的构造. 中国科学, A 辑, 2006, 36(2): 121 133

5 Chern S S, Shen Z. Riemann-Finsler Geometry. Singapore: World Scientific, 2005

6 Shen Z. Landsberg curvature, $S$-curvature and Riemann curvature. In: Bao D, Bryant R L, Chern S S, et al, eds. A Sampler of Riemann-Finsler Geometry. MSRI Series, Vol 50. Cambridge: Cambridge University Press, 2004. 303 355

7 Kitayama M, Azuma M, Matsumoto M. On Finsler spaces with $(\alpha, \beta)$-metric. Regularity, geodesics and main scalar. J of Hokkaido Univ of Education (Section II A), 1995, 46(1): $1 \sim 10$

8 Matsumoto M. Finsler spaces with $(\alpha, \beta)$-metric of Douglas type. Tensor N S, 1998, 60: 123 134 